\title{
Calcium oxalate crystal growth modification; investigations with confocal Raman microscopy
}

\author{
Calum J. McMulkin 1 , Massimiliano Massi ${ }_{1}$, Franca Jones 1. \\ ${ }_{1}$ Curtin Universitry of Technology, Department of Chemistry, GPO Box U1987, Perth, WA, Australia 6845. \\ E-mail: callum.mcmulkin@postgrad.curtin.edu.au; phone: +61 892669753
}

\begin{abstract}
Confocal Raman Microscopy (CRM) in combination with a photophysical investigation has been employed to give insight into the interaction between calcium oxalate monohydrate (COM) and a series of tetrazole containing crystal growth modifier's (CGM's), in conjunction with characterisation of morphological changes using scanning electron and optical microscopy. The tetrazole CGM's were found to interact by surface adsorption with minimal morphological changes to the COM crystals however, significant interactions via chemisorption were observed; it was discovered that the chemisorption is sufficiently strong for aggregation of the tetrazole species to occur within the crystal during crystallisation.
\end{abstract}

\section{Key words}

Biocrystallization, Crystal morphology, Optical microscopy, Growth from solutions, Calcium compounds.

\section{Introduction}

The formation of stones in the kidney, bladder or urinary tract $^{1}$ (urolithiasis) are undesirable biological crystallisation events that can occur within the human body; in particular, kidney stone (renal calculi) formation is very common, with 1 in 10 people affected in their lifetime ${ }^{2}$. The most common type of human kidney stones are calcium containing stones, the major constituent of which is $\operatorname{COM}\left(\mathrm{CaC}_{2} \mathrm{O}_{4} \cdot \mathrm{H}_{2} \mathrm{O}\right)$ at $70 \%{ }^{3}$. COM is the thermodynamically stable phase of calcium with oxalate at room temperature but has two other meta-stable phases, the dihydrate and the trihydrate, with the dihydrate also being commonly present in human kidney stones while the trihydrate is uncommon. Currently, few medicines used for the treatment of kidney stones do not have risks inherent with their use and thus surgical removal and other techniques including extracorporeal shock-wave lithotripsy (ESWL) and medical expulsive therapy (MET) are used to combat the already grown calculi ${ }^{4}$. Research in the field, therefore, focuses on kidney stone prevention employing crystal growth modifiers (CGM's) as a means of controlling kidney stone formation. Those inflicted with the condition would benefit greatly from the development of therapeutics that hinder the formation of nuclei or suppresses their growth. Crystal growth modifiers recently studied include but are not limited to carboxylic acids, calixarenes, proteins and polymers ${ }^{5-8}$.

Recently researchers have been utilising the bioisostere nature of carboxylic acids and tetrazoles ${ }^{9-13}$ to investigate the effect the tetrazole moiety has in medicine, and more recently in the field of crystal growth modification. Before our group started investigating tetrazoles as modifiers, they had not been used as crystal growth modifiers; now crystal systems have been investigated including, barium sulfate, calcium carbonate ${ }^{14}$, and calcium oxalate ${ }^{15}$, all of which were discovered to be inhibited by the presence of tetrazoles during crystallisation. The tetrazole species used in this study are shown in Figure 1. This study, in which we present our preliminary results, is a continuation and expansion of the work mentioned above to try to elucidate the mechanism of interaction between COM crystals and the tetrazole species during crystallisation. We have utilised the capabilities of Confocal Raman Microscopy (CRM) and a photophysical investigation to provide insight into the mechanism of COM modification in the presence of a series of tetrazoles which have varying degrees of impact as CGM's, the morphology changes were monitored by optical microscopy and SEM.<smiles>[R]c1nnn[nH]1</smiles><smiles>[R]=[Ni]</smiles>

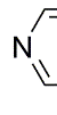<smiles>Cc1cccnc1</smiles><smiles>[R]c1ccc(-c2nnn[nH]2)cc1</smiles>

\section{TzpPyrH}

TzmPyrH

TzoPyrH

\section{TzPyzH}

Figure 1. Tetrazoles species structures used as CGM's (named from top left down to top right down) 5-(4-Pyridyl)-1H-tetrazole (TzpPyrH, 6.84 mM), 5-(3-Pyridyl)-1H-tetrazole (TzmPyrH, 6.84 mM), 5-(2-Pyridyl)-1H-tetrazole (TzoPyrH, $6.84 \mathrm{mM})$, 5-(3-Pyrizine)1H-tetrazole (TzPyzH, 6.88 mM), 5-(4-Bromophenyl)-1H-tetrazole (TBrPhH, $4.51 \quad \mathrm{mM}), \quad$ 5-(4-methoxyphenyl)-1H-tetrazole (TzMeoxPhH, 5.78 mM). 5-(4-phenol)-1H-tetrazole (TzPhoH, 6.22 $\mathrm{mM})$.

\section{Results and discussion}

CRM investigation of COM in the presence of CGM's.

As has been previously reported ${ }^{15}$, tetrazoles have been found to change the morphology of COM crystals but seemingly through different mechanisms of interaction than their carboxylic acid equivalent counter-parts. 
This manuscript looks at understanding the interactions with similarly sized molecules as shown in Figure 1, with emphasis on the adsorption of the molecules to the crystal. The tetrazole species TzmeoxPh- will be used as the exemplary CGM throughout this manuscript for clarity.

Firstly, the morphology of control crystals for our system are shown in Figure 2A, mostly twinned COM crystals between 10 and $15 \mu \mathrm{m}$ long, $\sim 5 \mu \mathrm{m}$ wide, $\sim 8 \mu \mathrm{m}$ deep. Upon addition of all of the CGM's above in Figure 1, reduction in the number of crystals is evident with some additives suppressing nucleation to such significant levels few particles could be found to analyse. The most potent inhibitors were TzoPyr-, TzBrPh- and TzmeoxPh', each showing the greatest reduction of nuclei formed at their given concentrations; see supplementary Figure S1†-S3† for SEM images of the series and Table $\mathrm{S} 1 \dagger$ for quantitative data for the series. Fig $\mathrm{S} 3 \dagger$ illustrates the significant drop in the amount of particles due to the presence of many of the CGM's. However, due to the reduction in the number of nuclei during crystallisation, the particles present are subsequently larger in size than that of the control COM crystals. Interestingly single crystals now dominate instead of the more common twinned crystals shown in the control example. Negligible morphological changes were seen with this series of tetrazoles however, significant impacts on the nucleation of the crystals was clearly evidenced (Table S1†).

The Raman spectra presented in Figure 3 correspond to the COM formed under control conditions and in the presence of the TzmeoxPh species. In the spectra provided the evidence of an interaction between COM and the tetrazole will be elucidated. Figure $3 \mathrm{~A}$, represents the Raman signal collected at the surface of a control COM crystal, it shows the characteristic peaks given for COM from the literature ${ }^{16}$ at $\sim 1470 \mathrm{~cm}^{-1}, 1490 \mathrm{~cm}^{-1}$ and $\sim 500 \mathrm{~cm}^{-1}$. The doublet at $1470 \mathrm{~cm}^{-1}$ and $1490 \mathrm{~cm}^{-1}$ are characteristic of the monohydrate phase and not of the dihydrate or trihydrate phase for calcium oxalate. Figure 3B, displays a typical Raman spectrum for COM grown in the presence of our tetrazole species; in this case, TzmeoxPh- The characteristic peaks for COM are still present but the signals are diminished in intensity due to the lowering of laser intensity to avoid exciting the tetrazole species (which fluoresces quite strongly in the regions where the characteristic peaks are visible). Figure 3B's spectrum was taken at the surface of the crystal, which in contrast to Figure $3 \mathrm{C}$ illustrates the capabilities of the CRM; this spectrum was taken a few micrometres within the crystal using the confocal ability of the instrument and allows for the observation of the fluorescence and Raman signals from the tetrazole at subsurface layers. The presence of these Raman vibrational frequencies indicates we have tetrazole species present within the crystals and not solely adsorbed to the surface, otherwise we would have observed a similar signal present for Figure 3B. For all the Raman spectra collected containing CGM's, the main signals for the tetrazole species are found at $\sim 1340 \mathrm{~cm}^{-1}$ and $1600 \mathrm{~cm}^{-1}$, these peaks are believed to belong to either the $\mathrm{C}-\mathrm{N}$ or $\mathrm{N}-\mathrm{N}$ stretching frequencies of the tetrazole, see Figure $3 \mathrm{~S} \dagger$ for the Raman of TzmeoxPhH only, which due to the process of adsorption has characteristic peaks that are different in position to those shown here. As mentioned above, the laser intensity was reduced in order to see the structure of these bands and

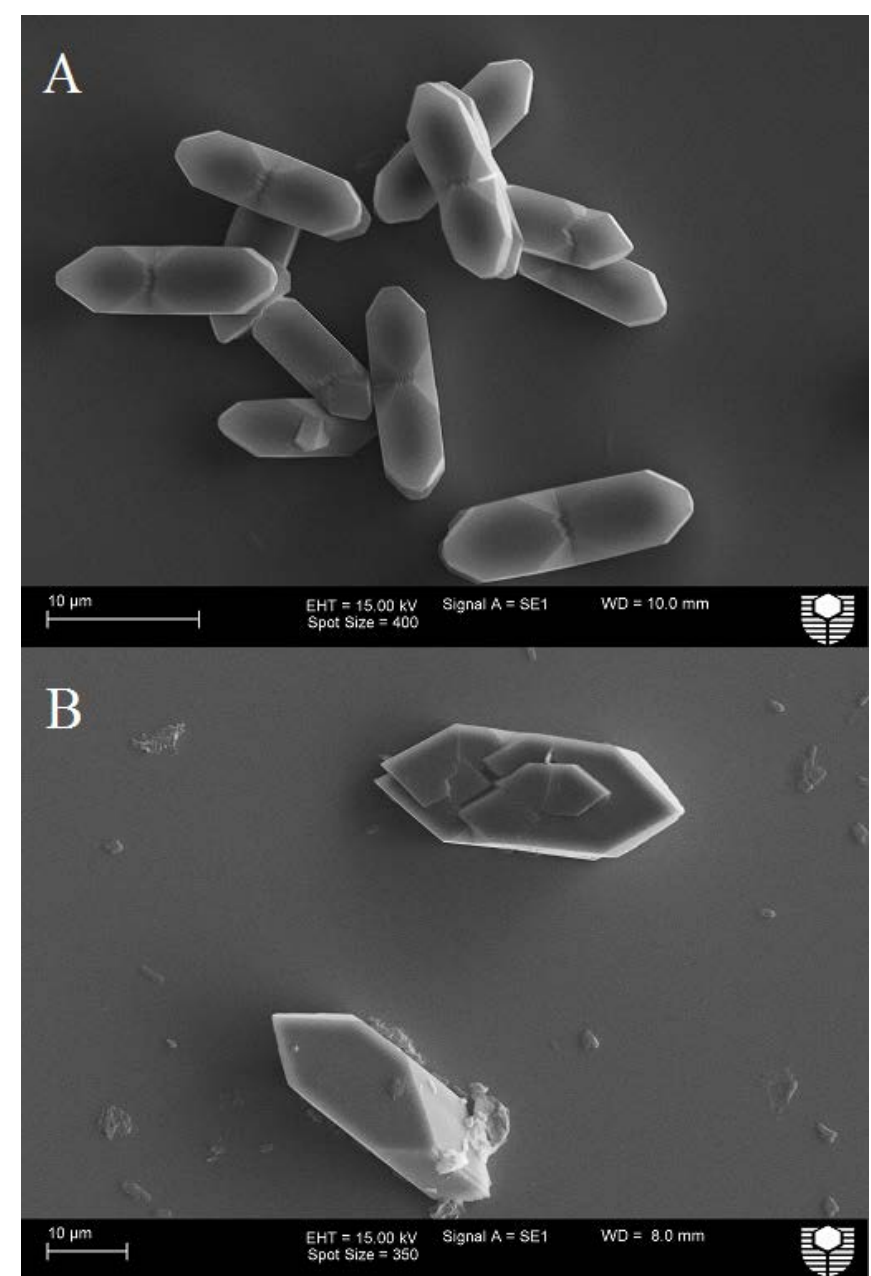

Figure 2. SEM images of COM crystals, A) Control crystals, B) Crystals grown in the presence of TzmeoxPh ${ }^{-}(5.78 \mathrm{mM})$.

upon increasing the intensity of the laser, the fluorescent properties of the tetrazole species mask the structured peaks for both the tetrazole and COM. Figure 3D fully exemplifies why CRM has been selected as the technique used to investigate the presence of the tetrazole species within the COM crystals. As presented, the $\mathrm{Y}$-axis indicates the intensity of the chosen Raman signals monitored during collection of the spectrum against the distance through the crystal (in micrometres, on the X-axis). CRM allowed us to plot the intensity of the characteristic signal for the COM (blue trace $-1470 \mathrm{~cm}^{-1}$ ) and the tetrazole (red trace - 1340 $\mathrm{cm}^{-1}$ ) allowing for a comparison between the two species' intensities with respect to the position inside the COM crystal. This allows us to determine, with confidence, the location of the tetrazole species within the crystal itself. The tetrazole species have been found to reside closer to the surface of the COM crystals and relatively less found towards the centre of the crystal, the working hypothesis for this observation is that at the early stages of crystallisation i) the crystal faces of COM are not well developed for the tetrazole to adsorb onto specific faces and ii) during this time competition between the oxalate and tetrazole species occurs, which favours the oxalate species due to the initial high supersaturation. The interaction between the tetrazole and crystal is believed to occur via adsorption to the surface and subsequent growth of the COM around the tetrazole species resulting in what is observed in our system; the tetrazole species being included into the crystal itself as verified by our confocal Raman spectroscopy. 

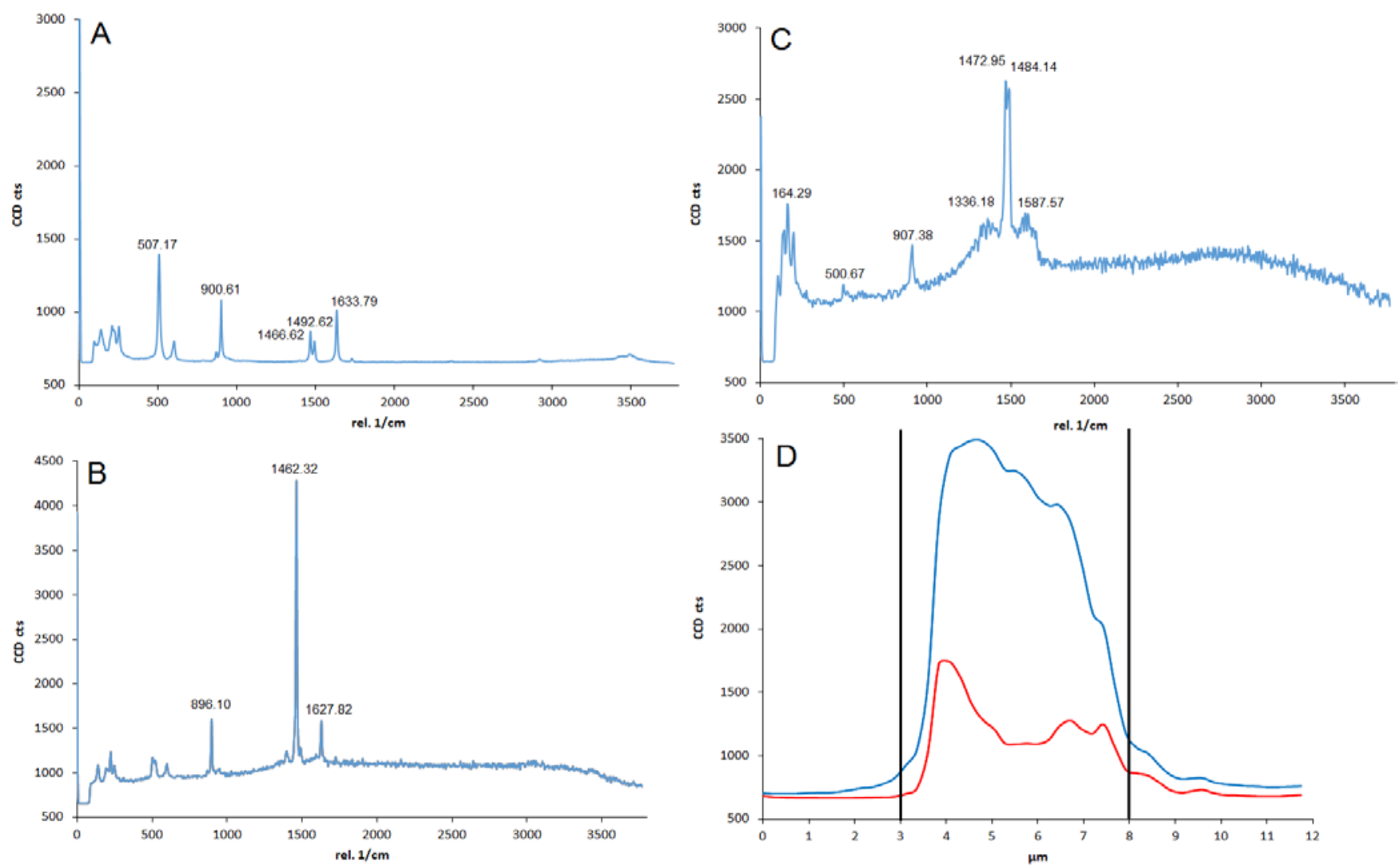

Figure 3. Confocal Raman Spectra A) At surface of control COM crystals, B) Of COM crystals grown in the presence of TzmeoxPh- (5.78 $\mathrm{mM}^{-1}$ ), C) Raman spectrum taken within COM crystals grown in the presence of TzmeoxPh- (5.78 mM), D) Intensity of main characteristic Raman signals against the position each individual confocal Raman spectrum was taken, blue trace belongs to the COM crystals characteristic peak $\left(\sim 1470 \mathrm{~cm}^{-1}\right)$, while the red trace belongs to the tetrazole characteristic peak $\left(\sim 1340 \mathrm{~cm}^{-1}\right)$, the black vertical lines represent the location of the crystal surfaces, at $\sim 3 \mu \mathrm{m}$ lies the top surface and at $\sim 8 \mu \mathrm{m}$ lies the bottom of the crystal. Refer to text for in-depth description of each image in Figure 3.
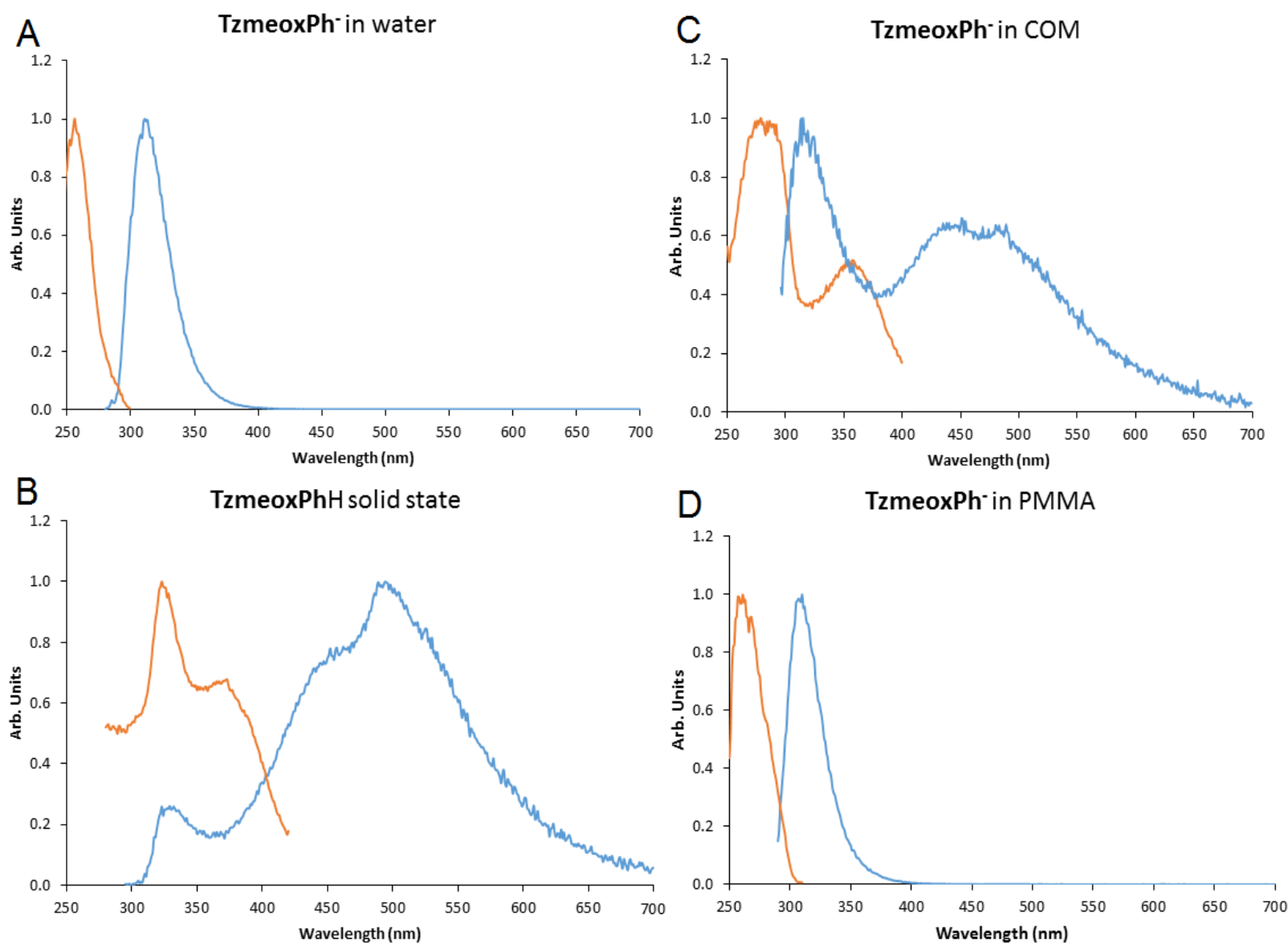

Figure 4. Emission and excitation profiles of TzmeoxPhH in four different states (orange trace corresponds to excitation, blue to emission). A) TzmeoxPh- $^{-}\left(10^{-6} \mathrm{M}\right)$ in water, B) TzmeoxPhH solid state profile C) COM crystals profile grown in the presence of $\mathrm{TzmeoxPh}^{-}$and $\mathrm{D}$ ) Profile of TzmeoxPh- dispersed in a PMMA polymer. 


\section{Photophysical investigation of tetrazole species within COM crystals}

As mentioned above, what was common across the series of tetrazoles was the presence of very fluorescent Raman signals when the crystals were not washed before Raman analysis see Figure $4 \mathrm{~S}+$. This led us to subsequently wash the crystals with water. In all cases this removed the surface Raman signal we were seeing, which corresponds to the removal of surface adsorbed (physisorbed) tetrazolate species. What was more surprising was when we used the confocal properties of the Raman spectrometer to probe the crystal we found that significant Raman signals from tetrazole species were still present. This structured Raman signal is proposed to be due to chemisorbed tetrazolate species within the COM crystal.

The presence of the tetrazole species within the crystals was investigated further through a photophysical investigation (as tetrazoles are known to be emissive) and the results are as follows: during the adsorption to the surface of the COM crystals, the tetrazole molecules aggregate together and this process results in not only the Raman signals we report above, but also the emission and excitation profiles seen in Figure 4. The excitation and emission spectra are presented in each image $A$ through $D$ for the tetrazole TzmeoxPhH in four different environments. Figure 4A shows the tetrazole species alone in an aqueous solution with one emission band, that of the $\pi \pi^{\star}$ transition, common for species with extended interannular conjugation. The solid state tetrazole provided the emission and excitation profiles shown in Figure $4 \mathrm{~B}$. It can clearly be seen that this is very similar to the emission profile seen when the COM crystals are grown in the presence of the tetrazole (Figure 4C). Minor differences in the intensity of the aggregated peaks and the $\pi \pi^{*}$ transition might be due to the different environments in which they are found. Additionally, upon exciting the COM crystals alone, we also determined that the signal seen in Figure $4 \mathrm{C}$ could not have come from COM as it does not emit at all, see Figure 5S†. Finally, to verify that the aggregated emission profile is not due to the difference between the tetrazole species in their tetrazolate state as opposed to the protonated state during crystallisation, the tetrazole species were dispersed in PMMA polymers in their protonated and deprotonated states and the results are shown for the tetrazolate species TzmeoxPh- in Figure 4D, while the protonated species is identical (Figure 6S†). Thus, in the COM crystal the tetrazole species is aggregated and the lack of an aggregated state between the protonated and deprotonated species verifies that the aggregation phenomenon is related to the interaction with COM crystals. This aggregation is seen in all of the tetrazole species investigated.

\section{Conclusion}

The photophysical investigation and exploration using CRM facilitated in probing the interactions between our tetrazole CGM's and COM crystals. It was also established that our tetrazole species change the behaviour of the COM crystals during crystallisation mainly through a reduction in the number of particles formed. More importantly, our research determined that the strong adsorption interactions are followed by aggregation of the tetrazole within the crystal itself during growth. This is a unique mechanism of interaction which still has many aspects to be fully understood, an AFM investigation has begun to further elucidate this mechanism. Understanding of all the interactions involved would be highly beneficial in determining the extent of the morphological impact the adsorption process has alone, with and without the aggregation occurring. Once fully understood, the interactions could allow us to control COM crystallisation by inclusion of inhibitors into the lattice during growth or further yet by "activating" the aggregated species enveloped inside the crystals for controlled dissolution.

\section{Acknowledgements}

The authors acknowledge the use of equipment and technical assistance of the Curtin University Electron Microscope Facility, which has been partially funded by the University, state and Commonwealth Governments.

\section{Notes and References}

$\dagger$ Electronic supplementary information (ESI) available: SEM images, Raman spectra of calcium oxalate particles in the presence of tetrazole, Raman of tetrazoles alone, experimental for synthesis of tetrazoles, growth of crystals, techniques used in analysis.

1. Pearle, M. S., Calhoun, E. A. \& Curhan, G. C. in Urological Diseases in America 283-319 (2007).

2. Moe, O. W. Kidney stones: pathophysiology and medical management. Lancet 367, 333-44 (2006).

3. Smith, L. The many roles of oxalate in nature. Trans. Am. Clin. Climatol. Assoc. 113, 1-20 (2002).

4. Moe, O. W., Pearle, M. S. \& Sakhaee, K. Pharmacotherapy of urolithiasis: evidence from clinical trials. Kidney Int. 79, 385-92 (2011).

5. Jones, F., Mocerino, M., Ogden, M. I., Oliveira, A. \& Parkinson, G. M. Bio-Inspired Calix[4]arene Additives for Crystal Growth Modification of Inorganic Materials. Cryst. Growth Des. 5, 2336-2343 (2005)

6. Bartlett, M. J. et al. Amino acid modified calixarenes as crystal growth modifiers. J. Mater. Sci. Technol. 21, 1-5 (2005).

7. Zhang, D., Qi, L., Ma, J. \& Cheng, H. Morphological Control of Calcium Oxalate Dihydrate by a Double-Hydrophilic Block Copolymer. Chem. Mater. 2450-2457 (2002).

8. Sayan, P., Sargut, S. T. \& Kıran, B. Calcium oxalate crystallization in the presence of amino acids, proteins and carboxylic acids. Cryst. Res. Technol. 44, 807-817 (2009).

9. Meanwell, N. a. Synopsis of some recent tactical application of bioisosteres in drug design. J. Med. Chem. 54, 2529-91 (2011).

10. Patani, G. a. \& LaVoie, E. J. Bioisosterism: A Rational Approach in Drug Design. Chem. Rev. 96, 3147-3176 (1996).

11. Ostrovskii, V. a., Trifonov, R. E. \& Popova, E. a. Medicinal chemistry of tetrazoles. Russ. Chem. Bull. 61, 768-780 (2012).

12. Herr, R. J. 5-Substituted-1H-tetrazoles as carboxylic acid isosteres: medicinal chemistry and synthetic methods. Bioorg. Med. Chem. 10, 3379-93 (2002).

13. Malik, M. A., Wani, M. Y., Al-Thabaiti, S. A. \& Shiekh, R. A. Tetrazoles as carboxylic acid isosteres: Chemistry and biology. J. Incl. Phenom. Macrocycl. Chem. 78, 15-37 (2014).

14. Massi, M., Ogden, M. I., Radomirovic, T. \& Jones, F. Tetrazoles: a new class of compound for crystallization modification. CrystEngComm 12, 4205 (2010).

15. McMulkin, C. J., Massi, M. \& Jones, F. Tetrazoles: calcium oxalate crystal growth modifiers. CrystEngComm 17, 26752681 (2015).

16. Edwards, H. G. M., Farwell, D. W. \& Jenkins, R. Vibrational Raman Spectroscopic Studies of Calcium Oxalate Monohydrate and Dihydrate in Lichen Encrustations on Renaissance Frescoes. J. raman Spectrosc. 23, 185-189 (1992). 